Membrane Biol., 4, 179; 1971). Thylakoids are disk-shaped vesicles found within the chloroplasts of green plants. The thylakoid membrane contains a light-driven electric generator, together with pigments which, when there is a change in the electric field across the membrane, respond with a shift of their spectral absorption bands. This electrochromic shift can be used as a linear indicator for the potential difference across the membrane. Junge and Schmid induced such a potential difference using a short flash of light and measured the resultant voltage, in arbitrary units, by observing the electrochromic shift. They also similarly followed the decay of voltage with time. This decay was attributable to the membrane discharging through its own resistance and from its time course they were able to calculate the current. By carrying out this procedure in the presence of valinomycin and potassium ions they were able to construct a current-voltage curve for the effect of valinomycin on the membrane. It is gratifying to note that this curve is of the same form as that found for artificial lipid membrane.

In case it is thought that this approach is restricted to the thylakoid membrane, it should be pointed out that Witt (Naturwissenschaften, 10, 514; 1969) and his co-workers have indicated that it ought to be possible to add the dye rhodamin-b to membranes and use its electrochromic shift as an index of electrical potential difference.

\section{NUCLEIC ACIDS}

\section{Antianticodon}

from our Molecular Biology Correspondent UNDER ordinary conditions a trinucleotide, such as ApApA, will not stick appreciably to the complementary trinucleotide, UpUpU. (Polymers are different: a centipede with a weak grip in each leg still hangs on hard.) On the other hand a tRNA will attach very strongly by its anticodon to a complementary trimer anchored on a ribosome, and even to a considerable extent to the free trimer. This inconvenient fact does not seem to have perturbed the voyeurs of the protein synthesis field one whit, but the more physically inclined workers in the area have been considerably exercised to account for it. The problem has now been illuminated by the work of Eisinger and his colleagues.

In a recent issue of Nature New Biology (231, 127; 1971), Eisinger, Feuer and Yamane introduced a superior new method of observing the binding of codon triplets to yeast phenylalanine tRNA. Where earlier workers had used the painful pro- cedure of equilibrium dialysis, they apply a spectroscopic method, which has the merits of simplicity, of not interfering with the system in the process of measurement- $a$ feature that removes a number of experimental uncertainties - of rapidity of execution and minute demands in material. It takes advantage of the presence of a unique, and strongly fluorescent base, $Y$, next to the anticodon. When an oligomer binds, the local change of environment is sufficient to engender an appreciable shift in fluorescence and this is made the basis of the binding measurements. By this means the thermodynamic parameters of the interaction of the RNA with its codon were determined, and it was not surprising to find a large unfavourable entropy term, arising from the conformational immobilization of the codon (as well, of course, as the association per se). Now it is generally supposed that the tRNA has a rigid tertiary structure, and it may be conjectured that the anticodon is designed for binding in that it is held in the conformation of one strand of a WatsonCrick helix. In such a case this strand at least of the complex would contribute no unfavourable entropy to the binding process, because it would need to undergo no further conformational freezing, and, in this, the system would differ from one involving two conformationally mobile species, such as trinucleotides.

Eisinger (Biochem. Biophys. Res. Commun., 43, 854 ; 1971) has now hit on a way of verifying this hypothesis. If a pair of transfer RNAs could be found, the anticodons of which are complementary to each other, then they should associate without any loss of conformational freedom, and the binding should be very much stronger. Phenylalanine and glutamic acid tRNA are such a pair. The fluorescent technique again played its part; indeed, probably because the glutamic acid tRNA has a thiouracil residue next to its anticodon, with a long-wavelength absorption band, to which excitation

\title{
Binding of Oestradiol in Uterine Nuclei
}

Brochemists interested in the actions of hormones at the molecular level can be broadly classified into two groups; those who are intent on cataloguing the various effects of these agents on their respective target organs and those whose work is directed towards a search for specific hormone receptor sites in cells. To some extent the former group has always made it an act of faith that all the metabolic consequences of the action of a particular hormone stem from a primary site of action within the cell, whereas those who study hormone receptors tend to assume that these molecules represent such primary sites. Probably the best understood example of the interaction between a hormone and its target cell is the case of oestrogen binding to the mammalian uterus. Studies by Jensen and his colleagues have established that specific receptors for oestradiol exist in both the cytoplasm and the nuclei of the uterine cells. A report by G. Shymala Harris in next Wednesday's Nature New Biology now suggests that the structure of the DNA is important for the binding of oestradiol to the chromatin fraction of the nucleus in the mammalian uterus.

Harris shows that the oestradiolreceptor complex from the nuclei of mouse uterus can be solubilized by the action of an enzyme which digests away the DNA. The receptor complex thus released is similar to that obtained when the nuclear chromatin is disrupted by high ionic strength. This result may mean either that the oestrogen-receptor complex in the nucleus is directly bound to the DNA or that the structural integrity of the DNA is essential for interaction of the complex with some other component of the chromatin. The implications of this finding are that the marked changes in uterine function resulting from uptake of oestradiol are initiated by direct interaction of the hormonereceptor complex with the genetic material.

The mere existence of "receptor" molecules which bind hormones does not prove, however, that the hormones exert their metabolic actions by such interactions. Indeed, it may be disadvantageous to the cell to possess a physiologically important site with a high affinity for a hormone because such tight binding could render a target organ relatively insensitive to variations in concentration of the hormone in the circulation. Furthermore, the concept that a hormone should act directly on an intracellular process may be a dangerous over-simplification of the subtleties of cellular regulation mechanisms. Biochemists who are familiar with the part played by cyclic AMP in the manifestation of the effects of a variety of hormones will appreciate that target tissues do not always possess physiologically relevant binding sites for the hormones within the cells. It is likely that a fuller understanding of the nature and significance of intracellular hormone receptors will come only from more extensive knowledge of the mechanisms of regulation in higher cells. 